\title{
Brain tumor stem cells: new targets for clinical treatments?
}

\author{
Patrizia Tunici, Ph.D., Dwain Irvin, Ph.D., M.P.H., Gentao Liu, Ph.D., \\ XiangPeng Yuan, Ph.D., Zeng Zhaohui, Ph.D., Hiushan Ng, M.S., and John S. Yu, M.D.
}

Maxine Dunitz Neurosurgical Institute, Los Angeles, California

\begin{abstract}
$\checkmark$ The observation of similarities between the self-renewal mechanisms of stem cells and cancer cells has led to the new concept of the cancer stem cell. In cases of leukemia, multiple myeloma, and breast cancer, cells with a high selfrenewal potential have been identified. Furthermore, investigators have shown these cells' ability to drive the formation and growth of the tumor. Brain tumors have also been reported to possess a subpopulation of cancer stemlike cells that have the ability to proliferate, self-renew, and be multipotent. When grafted into mice, these cells are also able to generate a tumor that recapitulates that of the patient from whom the cells were derived. The identification and characterization of this new category of cells call for new therapies capable of selectively targeting and killing these multifaceted cells.
\end{abstract}

\section{KeY WordS - brain neoplasm - cancer stem cell - CD133 • ABCG2 • side population}

I $\mathrm{T}$ was first reported in a study of leukemia that a population of neoplastic cells exhibit heterogeneity with respect to proliferation and differentiation. ${ }^{7}$ These stemlike cells, present within the leukemic population, possess a high capacity for self-renewal and proliferation that is not present in the majority of leukemic cells. ${ }^{6,24,50}$ An analysis of stem cells present in leukemia tumors demonstrated that their presence is necessary and sufficient to maintain the tumor population. ${ }^{36}$ Cancer stem cells associated with breast cancer $^{2}$ and acute myelogenous leukemia ${ }^{7}$ have been isolated and implanted into experimental animals to form new tumors; their success following implantation demonstrates that these cells are sufficient to generate tumors.

The study of normal neural stem cells can be applied to the brain tumor cell population by establishing a link between normal neurogenesis and brain tumorigenesis. ${ }^{34,40}$ Brain tumors are phenotypically heterogeneous because they are composed of cells expressing both markers of differentiation and nondifferentiation. Populations of proliferating tumor stem cells differentiate into more mature cell types that characterize the tumor. ${ }^{11,38,41,43}$

In 2003, Singh and colleagues ${ }^{44}$ reported the identification and purification of cancer stem cells from human brain tumors of different phenotypes. These cells possess a marked

Abbreviations used in this paper: $\mathrm{ABC}=$ adenosine triphosphate-binding cassette; $\mathrm{BCRP}=$ breast cancer resistance protein; $\mathrm{BTSC}=$ brain tumor stem cell; $\mathrm{DC}=$ dendritic cell; FACS = fluorescence-activated cell sorting; $\mathrm{GBM}=$ glioblastoma multiforme; $\mathrm{MDR}=$ multiple drug resistance; $\mathrm{MHC}=$ major histocompatibility complex; $\mathrm{SP}=$ side population. capacity for proliferation, self-renewal, and differentiation. The authors demonstrated that BTSCs have a high self-renewal capacity, which correlates with increased malignancy (such as that seen in a medulloblastoma compared with a low-grade glioma). Brain tumor stem cells were isolated by selecting cells expressing the neural stem cell surface antigen $\mathrm{CD} 133$. The authors demonstrated that $\mathrm{CD} 133^{+}$cells can differentiate in vitro into tumor cells that phenotypically resemble the patient's tumor. These tumor stem cells represent a fraction of cells constituting the tumor that are identified by their CD133 expression. The CD133 marker is a $120-k D$ five-transmembrane cell-surface protein. Previously known as a hematopoietic stem cell marker, ${ }^{31}$ CD133 was recently found to be a marker for normal human neural stem cells as well. ${ }^{51}$ Singh and colleagues were able to confirm the stem cell activity of $\mathrm{CD} 133^{+}$tumor cells by plating BTSCs at limiting dilutions. These investigators demonstrated that the self-renewal capacity of tumor cells was only present in the fraction of cells that expressed $\mathrm{CD} 33^{+}$. In addition, they found that the $\mathrm{CD} 133^{+}$population displayed a proliferative capacity not present in the $\mathrm{CD} 133^{-}$fraction.

Singh and colleagues ${ }^{44}$ suggest that an analysis of BTSCs may also provide a novel means for testing new therapeutic strategies for brain tumors that focus on the eradication of the tumor by targeting BTSCs. In fact, the presence of BTSCs in all the tumors examined may have important implications for understanding the underlying mechanisms of brain tumor dissemination.

To be considered a BTSC, a cell must be able to do the following: 1) generate clonally derived cells that form neu- 
rospheres; 2) self-renew and proliferate; 3 ) differentiate and express markers typical of brain cells (that is, those markers associated with astrocytes, oligodendrocytes, and neurons); and 4) generate tumors after in vivo transplantation in animal models that resemble the original tumor in donor patients.

Singh and associates ${ }^{45}$ reported that only $\mathrm{CD} 133^{+}$cells are able to generate tumors after having been grafted into the mouse brain, and these tumors resemble the donor patient's original tumor. An injection of only $100 \mathrm{CD} 133^{+}$ cells into the NOD-SCID mouse brain led to the growth of a tumor that could be serially transplanted and was histologically identical to the tumor harbored by the patient from whom these cells were derived. In contrast, an injection of $10^{5} \mathrm{CD} 133^{-}$cells did not generate tumor growth (Fig. 1). ${ }^{45}$ The cellular heterogeneity of brain tumors suggests that only a small fraction of cancer cells are able to regenerate a tumor and that targeting these cells could be an innovative approach to eradicating the tumor. ${ }^{1}$

The mechanism by which normal neural stem cells become malignant has not yet been elucidated. Hemmati and associates ${ }^{21}$ demonstrated that normal and tumor-derived spheres express bmi-1, even after the withdrawal of mitogen from the culture medium. The gene $b m i-l$ has been demonstrated to be important for self-renewal of both leukemic $^{27}$ and normal hematopoietic ${ }^{35}$ stem cells. Thus, the presence and persistent expression of bmi- 1 in tumor cells could indicate a greater capacity of these cells to self-renew.

Other groups have reported the isolation of tumor neural stem cells from human adult GBMs; these cells have the capacity to establish, sustain, and expand these tumors even after serial in vivo transplantation experiments. ${ }^{16}$ These cells also possess a self-renewal capacity, an extensive proliferation capacity, and multipotency. A gene expression profile was obtained for genes involved in the regulation of stem cells including the self-renewal homeobox gene $E m \times 2^{17}$ and tumor-related genes such as PTEN, $p 21, p 27, p 53$, and MDR $1 .{ }^{16}$

We previously reported that in adults, the GBM contains a subpopulation of cells that can self-renew and differentiate into mature cell types, recapitulating the complexity of a primary GBM. ${ }^{54}$ These cells have all the properties of a stem cell, including the ability to form neurospheres, express neural stem cell markers, and, when differentiated, form multilineage progeny. In addition, they can self-renew and proliferate to generate subspheres and different types of progeny. Spheres generated at clonal density can reform spheres after induction of differentiation and have genetic aberrations typical of brain tumors (Fig. 2). This is an important point that distinguishes cancer stem cells from normal stem cells. In fact, there has been some evidence suggesting that CD133, the marker used by other researchers to separate cancer stem cells from the main tumor cell population, may not be the appropriate marker for distinguishing normal stem cells from cancer stem cells. ${ }^{54}$

More remains to be elucidated on the role these cancer stem cells play in brain tumors. In particular, it has not yet been determined what cell is the origin of brain tumors. Two

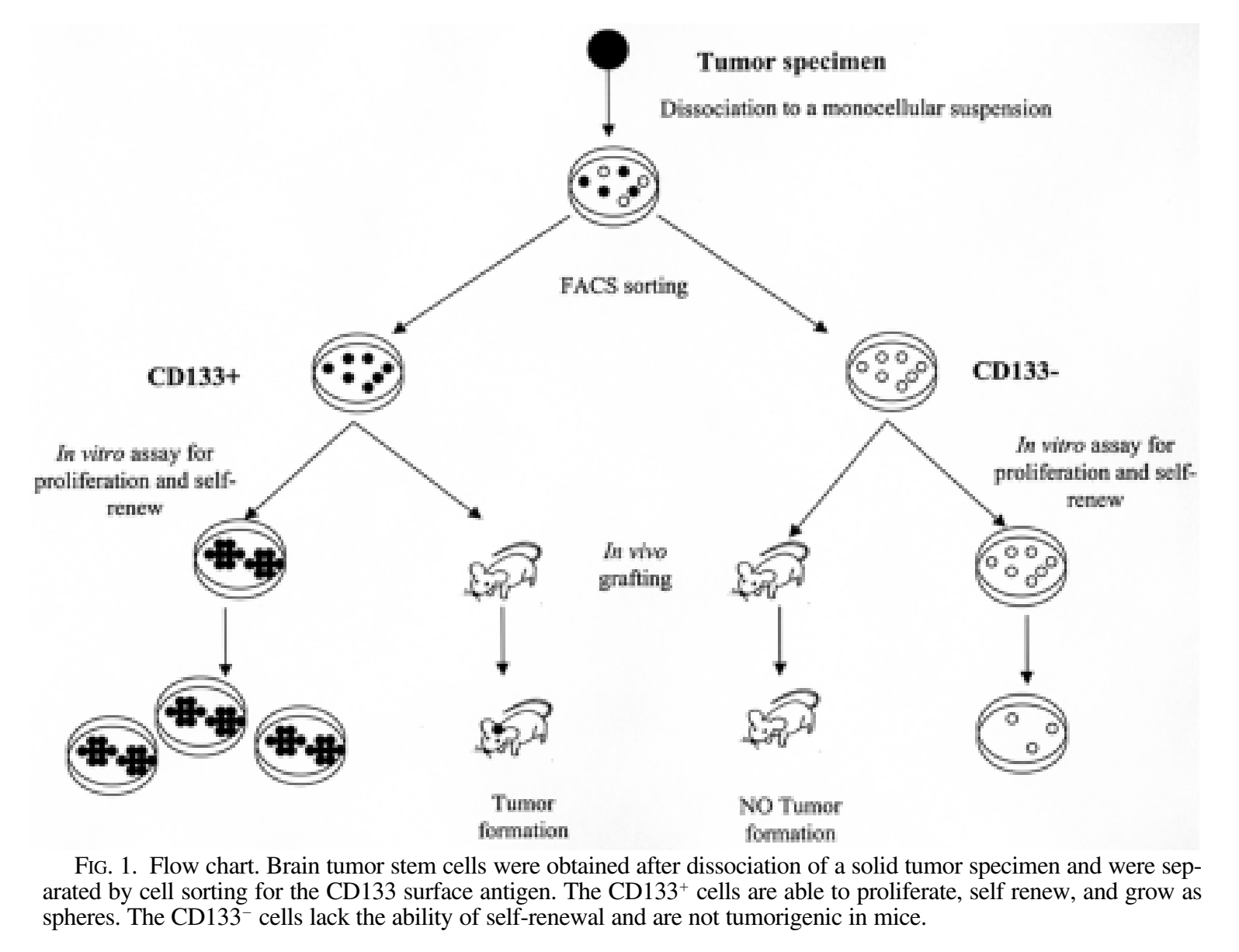




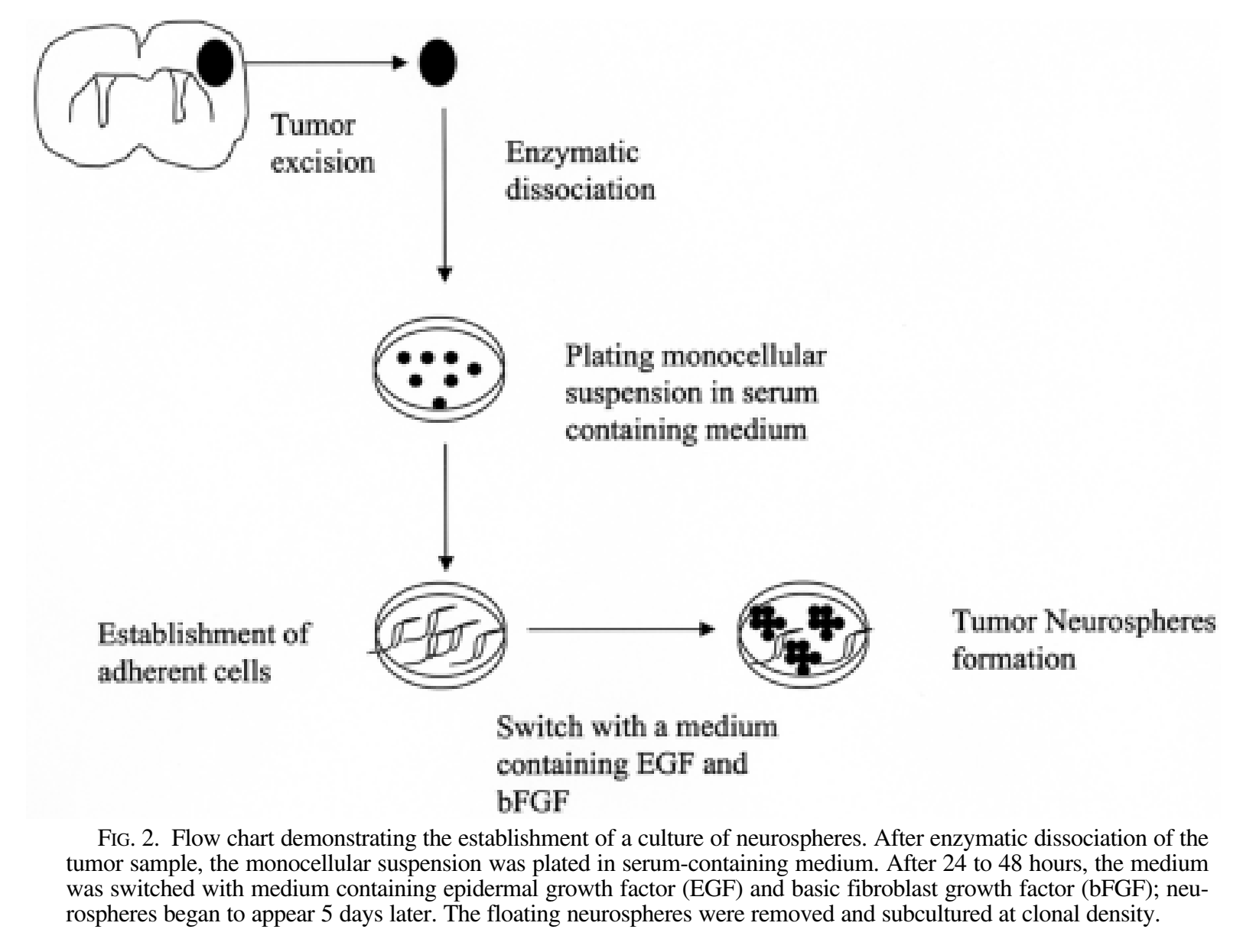

main hypotheses have been proposed: 1) brain tumors arise from the transformation of a normal stem cell or progenitor cell; and alternatively, 2) brain tumors arise from the dedifferentiation of a mature brain cell in response to genetic alterations. ${ }^{34,40}$ Many lines of evidence have suggested that brain tumors arise from normal stem cells that undergo genetic mutations; the similarities between normal stem cells and cancer stem cells regarding their self-renewal, cloning capacity, and multilineage differentiation corroborate this hypothesis ${ }^{34}$ In addition, the heterogeneity of brain tumors, which are composed of cells expressing more than one neural lineage phenotype, suggests an origin in a multipotent cell. It has also been reported that mutations in the mechanisms that regulate stem cell self-renewal (the sonic hedgehog pathway) can cause brain tumors. ${ }^{10,34}$ In vivo experiments have been performed to corroborate this hypothesis. Holland and collaborators ${ }^{23}$ demonstrated that undifferentiated neural precursors are more sensitive to transformation than differentiated cells. A recent review indicates the possibility that cancer stem cells originate from cell fusion or a horizontal gene-transfer process. ${ }^{5}$ The authors of that review report that cell fusion between somatic cells and stem cells might create genetic instability and cause cellular aneuploidy. Another mechanism suggested by the authors includes the possibility of a horizontal gene transfer. Mutations in somatic cells could lead to apoptosis and nuclear fragmentation: the fragmented DNA may be phagocytosed by other somatic cells, leading to nuclear reprogramming and the formation of new tumor cells. ${ }^{5}$ The possibility that end-terminal cells dedifferentiate into stemlike cells that acquire stemlike properties has not been formally ruled out.

The cancer stem cell hypothesis of the origin of tumor cells has been extended recently to a wide variety of tumors, including prostate cancer, ${ }^{9,29,49}$ bone sarcoma ${ }^{18}$ liver cancer, ${ }^{3}$ and melanoma. ${ }^{7}$ Collins and colleagues ${ }^{9}$ reported the isolation and characterization of a cancer stem cell population from human prostate tumors. These cells have a significant capacity to self-renew and are able to regenerate the phenotypically mixed population of nonclonogenic cells, which produces products of differentiated cells such as androgen receptor and prostatic acid phosphatase. The cancer stem cells derived from prostatic tumors have a CD $44^{+} \alpha 2$ $\beta 11^{\mathrm{hi}} \mathrm{CD} 133^{+}$phenotype, and these proteins were used to isolate cells from a series of prostatic tumors. ${ }^{9}$ In bone sarcomas, other investigators also identified a small population of cells with self-renewal properties. ${ }^{18}$ These cells were shown to be capable of forming "sarcospheres"- suspended spherical, clonal colonies that grow under serum-free conditions. These cells express activated STAT3 and some markers of embryonic stem cells, such as Oct-3/4 and Nanog, at a higher level of expression than that found in adherent cell cultures. A subpopulation of sarcoma cells expresses surface markers of mesenchymal cells such as Stro-1, CD105, and CD44. This finding extends the cancer stem cell hypothesis to cover tumors of mesenchymal origin..$^{18}$

Fang, et al. ${ }^{15}$ reported the presence of a subpopulation of 
cells in melanomas (melanoma spheroid cells) which grow as nonadherent spheres. These cells can differentiate into multilineage cells, such as melanocytes, adipocytes, osteocytes, and chondrocytes. These multipotent melanoma spheroid cells persist after serial cloning passages in vitro and transplantation in vivo, and are more tumorigenic than adherent cells after they have been implanted into mice. The stem cell population was enriched in CD20 $0^{+}$cells, a marker of hematopoietic stem cells. ${ }^{15}$ Cells that stained positively for this marker were separated using FACS techniques. Even if both $\mathrm{CD} 20^{+}$and $\mathrm{CD} 20^{-}$fractions display a similar proliferation capacity, $\mathrm{CD} 20^{+}$cells form bigger spheres. In one melanoma cell line (WM115), the fraction of cells that stained positively for CD20 was able to produce spheres, whereas the $\mathrm{CD} 20^{-}$cells remained single cells. In addition, when subjected to differentiation conditions, only the $\mathrm{CD} 20^{+}$fraction was able to generate multilineage cells. This indicates that melanoma spheroid stemlike cells enriched with CD20 ${ }^{+}$cells possess stem cell properties (the capacity for proliferation, self-renewal, and multipotent differentiation). ${ }^{15}$

Recently, it has been shown that ependymomas contain a rare population of cancer stem cells that display the radial glia phenotype and exhibit gene expression patterns that recapitulate those of radial glia cells in the corresponding region of the central nervous system. ${ }^{48}$ These cells are required and sufficient to propagate a tumor in transplanted mice. Ependymomas arising from different parts of the central nervous system contain different chromosomal abnormalities and possess a distinct gene expression signature. In particular, the Notch cell signaling pathway and the Hox family (known to regulate stem cell self-renewal) are upregulated in supratentorial and spinal ependymomas, respectively. ${ }^{48}$

The fact that it is possible to obtain cells with stem cell properties from different types of tumors corroborates the hypothesis that a small population of highly potent cells is present, in various degrees, in almost all tumors. Moreover, these cells are the "motor" that allows the tumor to grow. The presence of this population of cells can explain the recurrence of some brain tumors after chemotherapy, radiation therapy, and other current treatments. In fact, a few of these cells are sufficient to give rise to a new recurrent tumor. Cancer stem cells share another property with normal stem cells: they are able to migrate to different areas of the brain, thereby forming tumors far from the site of injection. In an experiment conducted by grafting these cells into mouse brains, tumors developed in a similar manner to those in humans. They mainly grew at the site of the injection, but were able to infiltrate the surrounding normal brain and produce tumors that extended into the contralateral hemisphere. Studies on the in vitro and in vivo behaviors of these cells may be useful to target not only differentiated tumor cells but also cancer stem cells.

\section{The Role Of Multiple Drug Transporters}

The cancer stem cell hypothesis provides novel insights into strategies to treat brain tumors. Recent data on the role of MDR transporters in the protection and self-renewal of normal and cancer stem cells may be used to identify pathways that can be specifically targeted. ${ }^{1}$ Since the develop- ment of anticancer drug therapies, it has been reported that some cancer cells demonstrate resistance to chemotherapy through the action of a group of membrane proteins that are able to extrude cytotoxic molecules and maintain an intracellular drug concentration below a cell-killing threshold. The discovery of the MDR family of genes (MDRl and $M D R 2$ ) allows us to understand more fully the mechanisms involved in the various responses of cells to treatment. ${ }^{12}$

Multiple drug transporters belong to a superfamily of $\mathrm{ABC}$ proteins located in the plasma membrane of different cellular organelles. ${ }^{19}$ These proteins mediate the transport of various molecules across the membrane and present a wide variety of chemical structures. In some instances, $\mathrm{ABC}$ proteins facilitate the transport of inorganic ions. Others pump organic molecules such as lipids, bile acids, glutathione, and short peptides. Most of the $\mathrm{ABC}$ proteins use adenosine triphosphate hydrolysis energy for this transport activity, while others form specific membrane channels. ${ }^{19}$

All of the ABC proteins share the ability to recognize and translocate a wide number of molecules, and additionally each transporter can efflux specific compounds. The first member of this family to be discovered was MDR-1 (also known as ABCB1), but two other members have been identified as playing a role in the multidrug resistance of the tumors: the multidrug resistance protein 1 (also known as ABCC1) and BCRP (also known as ABCG2). ${ }^{14,19}$

The BCRP is a half transporter consisting of only one nucleotide-binding domain and one membrane-spanning domain. It may function as a homodimer or homotetramer (Fig. 3). Overexpression of BCRP is associated with high levels of resistance to a variety of anticancer agents, including anthracyclines, mitoxantrone, and camptothecins by enhancing drug efflux. ${ }^{13,30}$ The expression of BCRP has been detected in a wide variety of hematological malignancies and solid tumors, underlying its role in the clinical drug resistance of tumors. ${ }^{39}$ The BCRP is highly expressed in the placenta, the apical membrane of the epithelium, the small intestine, the liver canalicular membrane, and the luminal surface of the endothelial cells of human brain microvessels. ${ }^{13}$ Zhou and coworkers ${ }^{55}$ demonstrated that ABCG2 is expressed in stem cells from a wide variety of sources. They found that the expression of ABCG2 is strictly correlated with SPs of cells from different sources (murine bone marrow, skeletal muscle, and cultured embryonic stem cells). The SP is defined as the population of cells able to efflux the Hoechst 33342 dye; it is rich in highly repopulating cells..$^{25}$ The SP was first demonstrated in hematopoietic stem cells of different species and in murine skeletal muscle and neurospheres derived from embryonic mice. ${ }^{8} \mathrm{An}$ SP was also found in various cancer cell lines including the C6 rat glioma, MCF7 breast cancer, B104 neuroblastoma, and HeLa adenocarcinoma cell lines. ${ }^{26}$ In C6 cells, the SP separated by FACS could generate both SP and non-SP cells in culture and form metastatic tumors in nude mice, but the non-SP cells could not. Therefore, the C6 SP contains cells with the characteristics of both normal stem cells and cancer stem cells. The persistence of SP cells in cancer cell lines cultured in serum-containing medium for decades suggests that the SP may be a general source of cancer stem cells. ${ }^{26,42}$

The correlation between SP cells and ABCG2-expressing cells appears to be controversial. Patrawala and colleagues ${ }^{37}$ reported that SP cells possess some intrinsic stem cell char- 


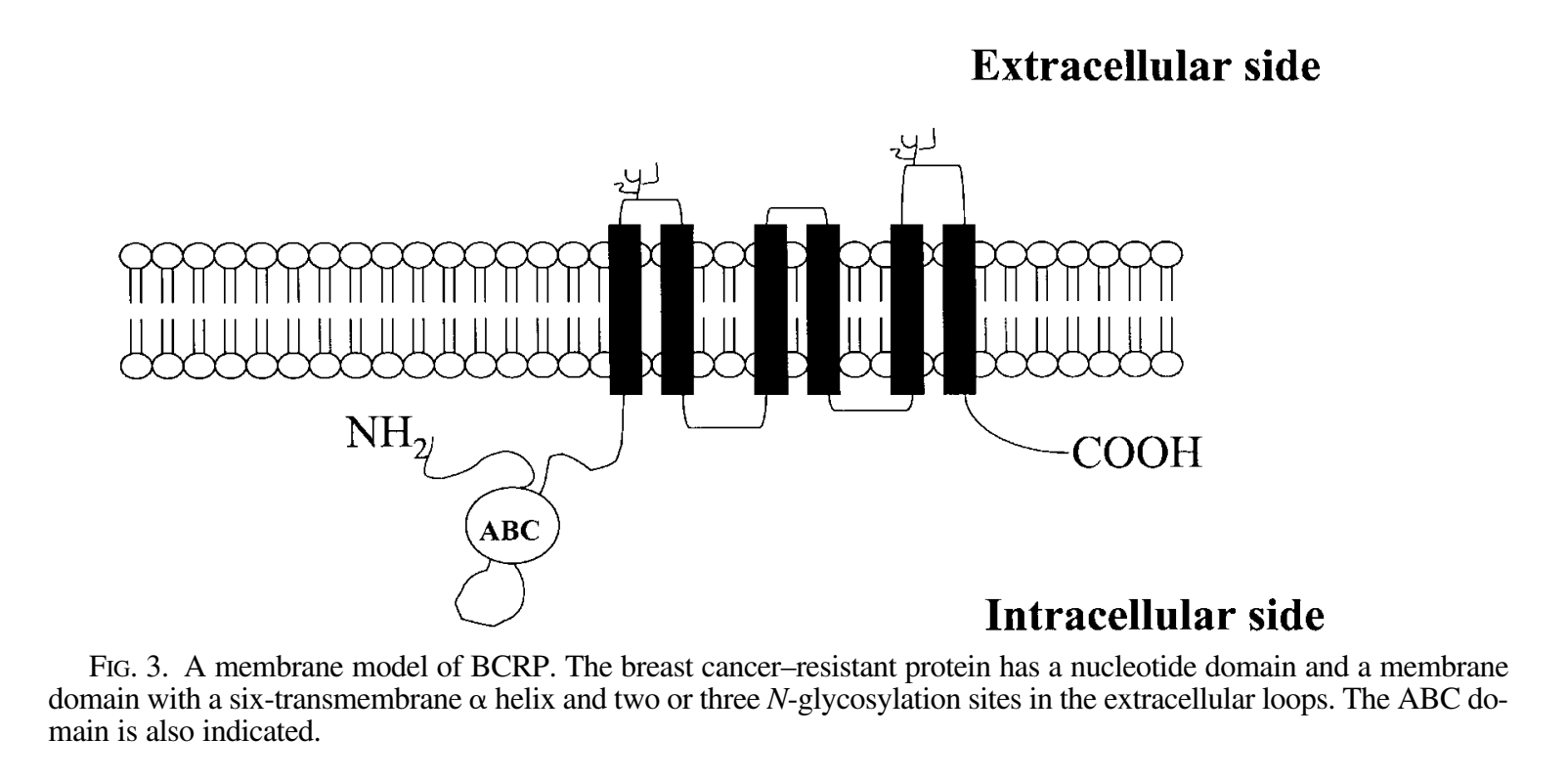

acteristics: they are able to generate both SP and non-SP cells, can be transplanted and generate tumors, express "stemness" genes such as Notchl and $\beta$-catenin, and demonstrate increased ABCG2 expression compared with nonSP cells. Nevertheless, although SP cells in vivo are more tumorigenic than non-SP cells, $\mathrm{ABCG} 2^{+}$purified cells display the same tumorigenicity as ABCG2- cells. The authors also demonstrated that $\mathrm{ABCG} 2^{-}$cells form larger and more numerous clones in long-term clonal analyses and express several "stemness" genes.

The SP cells also have been shown to be very heterogeneous. In fact, the SP detected in cancer stem cells contains a wide variety of cells, some of which express ABCG2. The presence of this subpopulation of cells can explain the increased expression of $\mathrm{ABCG} 2$ in the SP, but the higher tumorigenicity associated with the SP may also be conferred by the combined effects of other subpopulations of cells present in the SP. It could be possible that other adenosine triphosphate-binding proteins, such as MDR-1, MRP1 , and ABCA2, expressed in SP cells may play a role in conferring chemoresistance to this population of cells. ${ }^{37}$

\section{How To Target Cancer Stem Cells}

The presence and high expression of $\mathrm{ABC}$ transporter proteins in cancer stem cells may explain why common treatments, particularly chemotherapy, are not sufficient to kill the tumor. In fact, despite the continuous development of new chemotherapeutic agents, brain tumors remain resistant to therapy. Currently, the life span in patients harboring these lesions reaches 24 months in approximately 5\% of cases. Therefore, novel therapies must be developed. In particular, immunotherapy with vaccination of DCs opens a new way to target infiltrating brain tumors. Dendritic cells are antigen-presenting cells that stimulate the naïve immune system and play a role in maintaining self-tolerance. ${ }^{47}$ Through the expression of high levels of MHC class I and class II molecules, adhesion and costimulatory molecules (CD40, CD45, CD80, and CD86), and stimulatory cyto- kines (IL-12, IL-15, and IL-18), DCs lead to an efficient priming of cytotoxic $\mathrm{T}$ cells and $\mathrm{CD} 4^{+} \mathrm{T}$ helper cells, thus inducing a specific and therapeutic immune response. ${ }^{46}$ In fact, cytotoxic T cells are believed to be the main cells involved in tumor rejection, because these cells can recognize antigens loaded onto MHC class I molecules, whereas $\mathrm{CD}^{+} \mathrm{T}$ cells are activated after MHC class II-restricted presentation of exogenous antigens by DCs. It has also been reported that DCs can prime a $\mathrm{CD}^{+} \mathrm{T}$ cell-mediated response against exogenous tumor antigens. ${ }^{22}$ Dendritic cells can be generated in culture both from peripheral blood $\mathrm{CD} 14^{+}$monocytic precursors and from proliferating $\mathrm{CD} 34^{+}$ progenitor cells by the addition of cytokines, including IL4, granulocyte-macrophage colony-stimulating factor, tumor necrosis factor- $\alpha$, c-kit, and the Flt-3 ligand. ${ }^{46}$

In vaccination studies, DCs in culture were loaded with different tumor-derived substrates such as specific tumorassociated peptides, tumor RNA and complementary DNA, tumor cell lysate, or apoptotic tumor cells. ${ }^{46}$

Many animal studies and clinical trials have shown that DC immunotherapy improves and prolongs the survival of tumor-bearing experimental animals or patients., ${ }^{4}, 20,28,32,33,52$ We performed a Phase I clinical trial in which we administered DC-based vaccines to patients with gliomas (Fig. 4). ${ }^{53}$ We pulsed DCs with autologous tumor lysate and gave the vaccine to nine patients with recurrent GBM, three patients with recurrent anaplastic astrocytoma, and two patients with newly diagnosed tumors (one GBM and one anaplastic astrocytoma). The results we obtained showed that this tumor lysate-pulsed DC vaccine is safe, with no evidence of side effect or autoimmune toxicity, and can prolong the survival of patients (133 weeks compared with 30 weeks in control patients).

Future vaccination therapies may be directed toward cancer stem cell lysates to improve and ameliorate the antigen-presenting DC response. In this case, the activated immune system can directly kill tumor stem cells as well as tumor daughter cells. Preclinical studies are now necessary to test this hypothesis. 


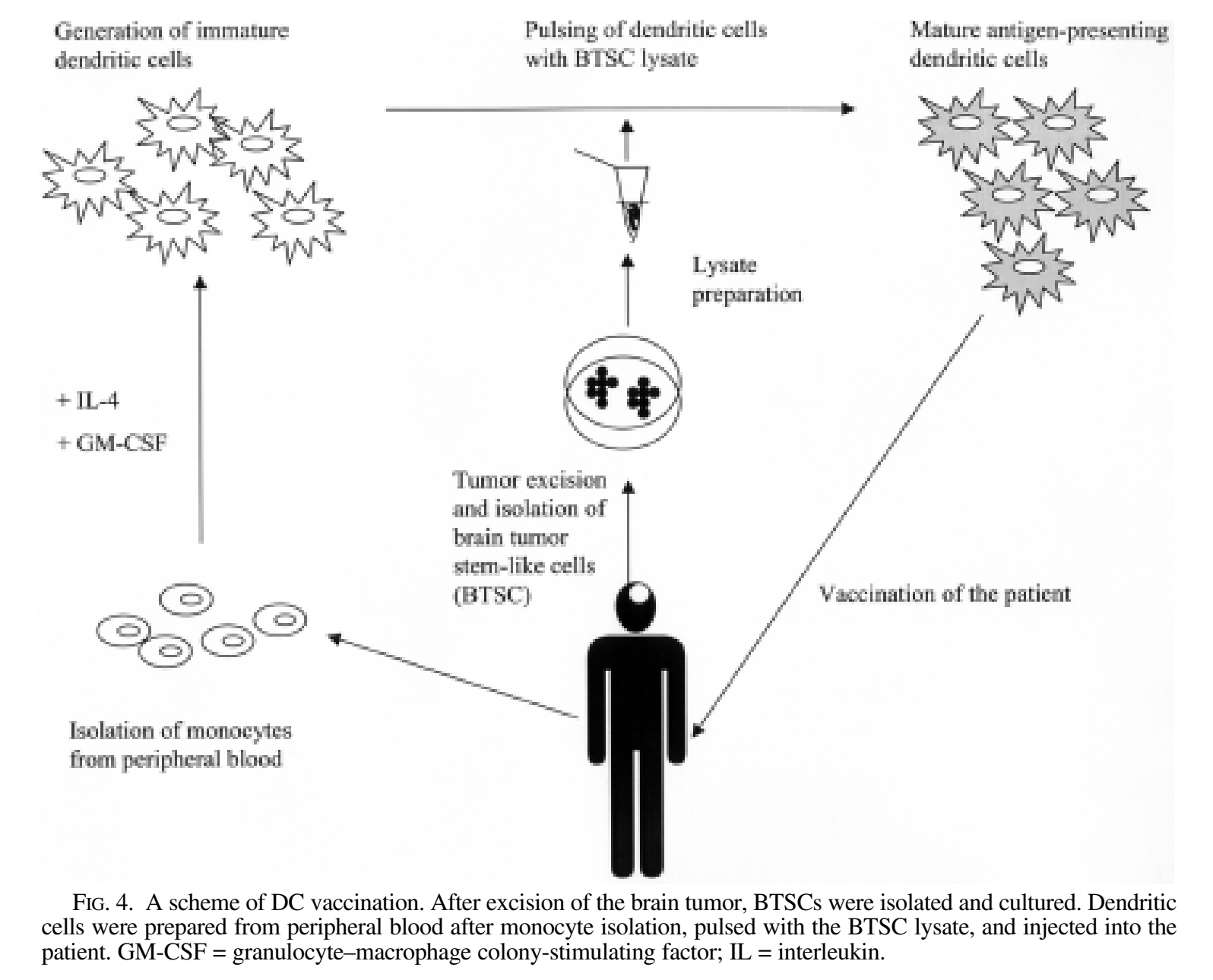

\section{Conclusions}

Although additional studies are necessary to provide us with a better understanding of the biology and behavior of tumor stemlike cells, it is evident that these cells represent a new target for future tumor therapies.

\section{References}

1. Al-Hajj M, Becker MW, Wicha M, et al: Therapeutic implications of cancer stem cells. Curr Opin Genet Dev 14:43-47, 2004

2. Al-Haij M, Wicha MS, Benito-Hernandez A, et al: Prospective identification of tumorigenic breast cancer cells. Proc Natl Acad Sci U S A 100:3983-3988, 2003

3. Alison MR, Lovell MJ: Liver cancer: the role of stem cells. Cell Prolif 38:407-421, 2005

4. Ashley DM, Faiola B, Nair S, et al: Bone marrow-generated dendritic cells pulsed with tumor extracts or tumor RNA induces antitumor immunity against central nervous system tumors. J Exp Med 186:1177-1182, 1997

5. Bjerkvig R, Tysnes BB, Aboody KS, et al: The origin of the cancer stem cell: current controversies and new insights. Nat Rev Cancer 5:899-904, 2005

6. Bonnet D: Cancer stem cells: lessons from leukaemia. Cell Prolif 38:357-361, 2005

7. Bonnet D, Dick JE: Human acute myeloid leukemia is organized as a hierarchy that originates from a primitive hematopoietic cell. Nat Med 3:730-737, 1997
8. Bunting $\mathrm{KD}$ : $\mathrm{ABC}$ transporters as phenotypic markers and functional regulators of stem cells. Stem Cells 20:11-20, 2002

9. Collins AT, Berry PA, Hyde C, et al: Prospective identification of tumorigenic prostate cancer stem cells. Cancer Res 65: 10946-10951, 2005

10. Dahmane N, Sánchez P, Gitton Y, et al: The sonic hedgehog-gli pathway regulates dorsal brain growth and tumorigenesis. Development 128:5201-5212, 2001

11. Dirks PB: Brain tumor stem cells. Biol Blood Marrow Transplant 11 (Suppl 2):12-13, 2005

12. Donnenberg VS, Donnenberg AD: Multiple drug resistance in cancer revisited: the cancer stem cell hypothesis. J Clin Pharmacol 45:872-877, 2005

13. Doyle LA, Ross DD: Multidrug resistance mediated by the breast cancer resistance protein BCRP (ABCG2). Oncogene 22:7340-7358, 2003

14. Ejendal KFK, Hrycyna CA: Multidrug resistance and cancer: the role of the human ABC Transporter ABCG2. Curr Protein Pept Sci 3:503-511, 2002

15. Fang D, Nguyen TK, Leishear K, et al: A tumorigenic subpopulation with stem cell properties in melanomas. Cancer Res 65:9328-9337, 2005

16. Galli R, Binda E, Orfanelli U, et al: Isolation and characterization of tumorigenic, stem-like neural precursors from human glioblastoma. Cancer Res 64:7011-7021, 2004

17. Galli R, Fiocco R, De Filippis L, et al: Emx2 regulates the proliferation of stem cells of the adult mammalian central nervous system. Development 129:1633-1644, 2002

18. Gibbs CP, Kukekov VG, Reith JD, et al: Stem-like cells in bone 
sarcomas: implications for tumorigenesis. Neoplasia 7:967-976, 2005

19. Gottesman MM, Fojo T, Bates SE: Multidrug resistance in cancer: role of ATP-dependent transporters. Nat Rev Cancer 2:48-58, 2002

20. Heimberger AB, Crotty LE, Archer GE, et al: Bone marrowderived dendritic cells pulsed with tumor homogenate induce immunity against syngeneic intracerebral glioma. J Neuroimmunol 103:16-25, 2000

21. Hemmati HD, Nakano I, Lazareff JA, et al: Cancerous stem cells can arise from pediatric brain tumors. Proc Natl Acad Sci U S A 100: 15178-15183, 2003

22. Hoffmann TK, Meidenbauer N, Dworacki G, et al: Generation of tumor-specific $\mathrm{T}$ lymphocytes by cross-priming with human dendritic cells ingesting apoptotic tumor cells. Cancer Res 60: 3542-3549, 2000

23. Holland EC, Celestino J, Dai C, et al: Combined activation of Ras and Akt in neural progenitors induces glioblastoma formation in mice. Nat Genet 25:55-57, 2000

24. Hope KJ, Jin L, Dick JE: Acute myeloid leukemia originates from a hierarchy of leukemic stem cell classes that differ in selfrenewal capacity. Nat Immunol 5:738-743, 2004

25. Kim M, Morshead CM: Distinct populations of forebrain neural stem and progenitor cells can be isolated using side-population analysis. J Neurosci 23:10703-10709, 2003

26. Kondo T, Setoguchi T, Taga T: Persistence of a small subpopulation of cancer stem-like cells in the C6 glioma cell line. Proc Natl Acad Sci U S A 101:781-786, 2004

27. Lessard J, Sauvageau G: Bmi-1 determines the proliferative capacity of normal and leukaemic stem cells. Nature 423:255-260, 2003

28. Liau LM, Black KL, Prins RM, et al: Treatment of intracranial gliomas with bone marrow-derived dendritic cells pulsed with tumor antigens. J Neurosurg 90:1115-1124, 1999

29. Maitland NJ, Collins A: A tumor stem cell hypothesis for the origins of prostate cancer. BJU Int 96:1219-1223, 2005

30. Mao Q, Unadkat JD: Role of the breast cancer resistance protein (ABCG2) in drug transport. AAPS J 7:E118-E133, 2005

31. Miraglia S, Godfrey W, Yin AH, et al: A novel five-transmembrane hematopoietic stem cell antigen: isolation, characterization, and molecular cloning. Blood 90:5013-5021, 1997

32. Ni HT, Spellman SR, Jean WC, et al: Immunization with dendritic cells pulsed with tumor extract increases survival of mice bearing intracranial gliomas. J Neurooncol 51:1-9, 2001

33. Okada H, Tahara H, Shurin MR, et al: Bone marrow-derived dendritic cells pulsed with a tumor-specific peptide elicit effective anti-tumor immunity against intracranial neoplasms. Int J Cancer 78:196-201, 1998

34. Pardal R, Clarke MF, Morrison SJ: Applying the principles of stem-cell biology to cancer. Nat Rev Cancer 3:895-902, 2003

35. Park IK, Qian D, Kiel M, et al: Bmi-1 is required for maintenance of adult self-renewing haematopoietic stem cells. Nature 423:302-305, 2003

36. Passegué E, Jamieson CHM, Ailles LE, et al: Normal and leukemic hematopoiesis: are leukemias a stem cell disorder or a reacquisition of stem cell characteristics? Proc Natl Acad Sci U S A 100: 11842-11849, 2003

37. Patrawala L, Calhoun T, Schneider-Broussard R, et al: Side population is enriched in tumorigenic, stem-like cancer cells, whereas $\mathrm{ABCG}^{+}$and $\mathrm{ABCG} 2^{-}$cancer cells are similarly tumorigenic. Cancer Res 65:6207-6219, 2005

38. Pilkington GJ: Cancer stem cells in the mammalian central nervous system. Cell Prolif 38:423-433, 2005

39. Plasschaert SLA, van der Kolk DM, de Bont ESJM, et al: The role of breast cancer resistance protein in acute lymphoblastic leukemia. Clin Cancer Res 9:5171-5177, 2003

40. Reya T, Morrison SJ, Clarke MF, et al: Stem cells, cancer, and cancer stem cells. Nature 414:105-111, 2001

41. Sanai N, Alvarez-Buylla A, Berger MS: Neural stem cells and the origin of gliomas. N Engl J Med 353:811-822, 2005

42. Setoguchi T, Taga T, Kondo T: Cancer stem cells persist in many cancer cell lines. Cell Cycle 3:414-415, 2004

43. Singh SK, Clarke ID, Hide T, et al: Cancer stem cells in nervous system tumors. Oncogene 23:7267-7273, 2004

44. Singh SK, Clarke ID, Terasaki M, et al: Identification of a cancer stem cell in human brain tumors. Cancer Res 63:5821-5828, 2003

45. Singh SK, Hawkins C, Clarke ID, et al: Identification of human brain tumor initiating cells. Nature 432:396-401, 2004

46. Soling A, Rainov NG: Dendritic cell therapy of primary brain tumors. Mol Med 7:659-667, 2001

47. Steinman RM, Turley S, Mellman I, et al: The induction of tolerance by dendritic cells that have captured apoptotic cells. J Exp Med 191:411-416, 2000

48. Taylor MD, Poppleton H, Fuller C, et al: Radial glia cells are candidate stem cells of ependymoma. Cancer Cell 8:323-335, 2005

49. Tokar EJ, Ancrile BB, Cunha GR, et al: Stem/progenitor and intermediate cell types and the origin of human prostate cancer. Differentiation 73:463-473, 2005

50. Tsai RYL: A molecular view of stem cell and cancer cell selfrenewal. Int J Biochem Cell Biol 36:684-694, 2004

51. Uchida N, Buck DW, He D, et al: Direct isolation of human central nervous system stem cells. Proc Natl Acad Sci U S A 97:14720-14725, 2000

52. Yamanaka R, Zullo SA, Tanaka R, et al: Enhancement of antitumor immune response in glioma models in mice by genetically modified dendritic cells pulsed with Semliki Forest virus-mediated complementary DNA. J Neurosurg 94:474-481, 2001

53. Yu JS, Liu G, Ying H, et al: Vaccination with tumor lysatepulsed dendritic cells elicits antigen-specific, cytotoxic T-cells in patients with malignant glioma. Cancer Res 64:4973-4979, 2004

54. Yuan X, Curtin J, Xiong Y, et al: Isolation of cancer stem cells from adult glioblastoma multiforme. Oncogene 23:9392-9400, 2004

55. Zhou S, Schuetz JD, Bunting KD, et al: The ABC transporter

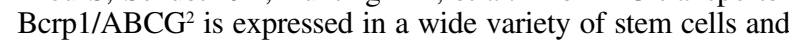
is a molecular determinant of the side-population phenotype. Nat Med 7:1028-1034, 2001

Manuscript received January 20, 2006.

Accepted in final form March 16, 2006.

Dr. Tunici is supported by ARIN (Associazione Italiana per le Ricerche Neurologiche, "Il Fondo di Gio").

Address reprint requests to: John S. Yu, M.D., Maxine Dunitz Neurosurgical Institute, Suite 800E East, 8631 West Third Street, Los Angeles, California 90048. email: John.Yu@cshs.org. 